\title{
Application de l'échographie en reproduction chez le zebu Goudali
}

\author{
Wéré PITALA ${ }^{1,5^{*}}$, Moussa ZONGO ${ }^{2}$, Hamidou BOLY ${ }^{3}$, Noelita Melo de SOUSA ${ }^{4}$, \\ Laya SAWADOGO ${ }^{2}$, Pascal LEROY ${ }^{4}$, Jean- François BECKERS ${ }^{4}$ et \\ Messanvi GBEASSOR ${ }^{5}$ \\ ${ }^{l}$ Ecole Supérieure d'Agronomie, Université de Lomé, BP 1515 Lomé, Togo. \\ ${ }^{2}$ UFR/SVT, Université de Ouagadougou, 03 BP 7021 Ouagadougou 03, Burkina Faso. \\ ${ }^{3}$ Institut du Développement Rural, Université Polytechnique de Bobo-Dioulasso, \\ 01 B.P. 1091 Bobo-Dioulasso, Burkina Faso. \\ ${ }^{4}$ Faculté de Médecine Vétérinaire. Université de, Liège, Bd. de Colonster, \\ 20, B43-4000 Sart tilman, Liège, Belgique. \\ ${ }^{5}$ Faculté des Sciences, Université de Lomé, BP 1515 Lomé, Togo. \\ *Auteur correspondant; E-mail : werepit@hotmail.com; Tel :002289094027
}

\section{RESUME}

Les travaux traitant de l'utilisation de l'échographie en reproduction animale ont été revus. Cette synthèse s'intéresse au contexte de l'application pratique de l'échographie en reproduction bovine, particulièrement le suivi de la croissance folliculaire jusqu'à l'ovulation après la synchronisation et l'induction des chaleurs, le diagnostic et le suivi de gestation chez les femelles zébus Goudali inséminées par la semence de Holstein. Toutes ces applications ont pu se réaliser grâce à l'échographie d'ultrasons (Pie Medical 6227, Philispweg 1, Maastricht, Pays-Bas). La détermination de la période d'ovulation après la fin d'un traitement de synchronisation de chaleur et de l'ovulation, le diagnostic précoce de gestation représentent des domaines prometteurs de l'utilisation de l'échographie. Cette technologie permettra d'améliorer nos connaissances en reproduction bovine et de rassurer les éleveurs des résultats de la pratique des biotechnologies de la reproduction. Malgré le coût élevé de la méthode échographique, elle prend de l'avance sur les autres méthodes de détermination des paramètres de la reproduction bovine (hormonologie et palpation rectale) car elle donne les résultats immédiats et observables par tous les acteurs présents sur place. L'échographie s'est avérée un outil fiable pour le suivi de la croissance folliculaire, la détermination de l'intervalle entre le début des chaleurs et l'ovulation et un outil diagnostique fiable dès le $28^{\text {ème }}$ jour post-insémination.

(c) 2012 International Formulae Group. All rights reserved.

Mots clés: Echographie, croissance folliculaire, diagnostic de gestation, bovins.

\section{INTRODUCTION}

Les impératifs économiques de l'élevage bovin obligent les éleveurs à optimiser le potentiel de production de leur troupeau par une réduction de l'intervalle entre vêlages et l'identification précoce des femelles non gestantes. A ce titre et parmi d'autres, la dynamique folliculaire et le diagnostic de gestation sont des activités essentielles dans une approche préventive du suivi de reproduction. Chez la vache, l'échographie est un examen complémentaire 
de la palpation transrectale, le premier examen durant lequel il est nécessaire de repérer les différentes structures en procédant de manière systématique avant l'échographie proprement dite. A la différence de la palpation, l'échographie présente les avantages de permettre avec une grande exactitude, de visualiser les structures ovariennes, d'évaluer le stade physiologique de l'utérus et de faire des diagnostics de gestations précoces (Mialot et al., 2002; Boly et al., 2003; Calais et Dreno, 2004 ; Francony, 2011). Ainsi, l'échographie bovine est devenue un outil de gestion de la reproduction et est utilisée à trois niveaux d'activités :

- Dans les programmes de recherche par les physiologistes ou les cliniciens spécialisés en reproduction ;

- pendant la sélection, la préparation et la surveillance des vaches donneuses et receveuses dans les programmes de transfert, le sexage d'embryons et pour la récolte d'ovocytes (Tainturier et al., 2003; Cros, 2005) ;

- en pratique quotidienne comme technique de diagnostic, notamment pour détecter les métrites post-partum, pour observer l'activité des follicules et des corps jaunes normaux ou pathologiques sur les ovaires et pour le diagnostic de gestation (DesCôteaux et al., 2006 ).

Comparativement aux protocoles de dosage de la progestérone et de la $\mathrm{LH}$, l'échographie présente l'avantage de donner sans délai des informations sur l'état physiologique des ovaires, de fournir une estimation correcte du nombre des follicules impliqués dans la croissance pré-ovulatoire et de pouvoir estimer le nombre de corps jaunes, synonyme du nombre d'ovulations. De même, l'échographie permet chez la vache une observation directe du produit de conception.

Cette synthèse vise à présenter les résultats issus des applications pratiques de l'échographie dans la caractérisation de la croissance folliculaire terminale au cours de cycles stimulés, dans le diagnostic, et le suivi de gestation chez les femelles zébus Goudali réalisée en zone péri-urbaine de
Ouagadougou.

\section{MATERIEL ET METHODES Matériel}

L'échographe utilisé est un appareil ultrasonore portable de modèle 200 Vet (Pie Medical 6227, Philispweg 1, Maastricht, Pays-Bas) équipé d'une sonde à balayage linéaire de fréquence de $6 \mathrm{MHz}$. Cet échographe a été relié à un ordinateur qui permet d'enregistrer les différents échotomogrammes (images échographiques) grâce à une carte Matrox (Matrox Graphics, Québec, Canada). Le matériel biologique a été constitué de quatre vingt quatre (84) femelles bovines de race zébu Goudali. Les gants protecteurs d'exploration transrectale à usage unique et le gel ont été utilisés.

\section{Méthodologie de l'examen échographie}

L'échographie est une technique d'investigation complémentaire qui permet de visualiser la structure, les contours et les rapports des organes internes pleins non calcifiés en utilisant leur capacité à absorber ou à réfléchir les ultrasons. Le signal transmis correspondant aux différentes structures rencontrées (écho) sera analysé par un ordinateur et restitué en temps réel sur l'écran. Le mot "échographie" de façon littérale signifie " graphe ou visualisation des échos ".

Chez les bovins, l'échographie est considérée comme un examen complémentaire. Avant d'échographier une vache, il est nécessaire de connaître entre autres les commératifs suivants: la date de vêlage, la date des chaleurs, la date de l'insémination artificielle ou de la monte. Les enregistrements des différentes images sur un ordinateur ont été possibles grâce à une carte graphique. La carte graphique a été connectée sur une sortie vidéo du moniteur de l'échographe. Les échotomogrammes les plus représentatifs ont été gelés pour effectuer les mensurations nécessaires puis enregistrés à l'ordinateur pour des analyses d'images plus affinées.

\section{Echographie des ovaires}

L'examen échographique des ovaires 
est réalisé par voie transrectale du fait que chez les bovins, la profondeur des organes génitaux ne permet pas leur visualisation par l'extérieur selon la technique décrite par Quirk et al. (1986) et par Pierson et Ginther (1988). Une évacuation complète du rectum est indispensable. Ceci permet une appréciation de la topographie des différents organes dans la filière pelvienne, leurs rapports et modifications anatomiques liées à un processus physiologique ou pathologique. La sonde enduite de gel de contact est introduite dans le rectum de la vache. La sonde est habituellement maintenue au moyen du pouce, de l'index et du majeur tandis que la manipulation de l'ovaire et son maintien contre la sonde est assuré par l'annulaire et le petit doigt. Les dimensions des follicules sont mesurées après gel de l'image afin de suivre leur croissance jusqu'à l'ovulation. Un diamètre folliculaire moyen est calculé entre le plus petit et le plus grand diamètre.

\section{Méthodologie de diagnostic de gestation}

Après l'évacuation de rectum comme précédemment et la localisation des cornes par palpation, la sonde est introduite dans le rectum. Celle-ci est posée sur la muqueuse du plancher du rectum. La progression de la sonde dans l'ampoule permet de visualiser les organes sous-jacents. On visualise tout d'abord la vessie, puis le col et le corps utérin en maintenant la sonde horizontale. En inclinant la sonde d'un côté, puis de l'autre, les cornes utérines sont successivement mises en évidence (Chastant-Maillard et al., 2002). Elles sont alors coupées longitudinalement par le faisceau d'ultrasons qui balaye toute la longueur des deux cornes utérines. Les éléments caractéristiques de la gestation recherchés sont:

- La présence d'un liquide utérin aqueux (zones complètement anéchogènes) correspondant aux liquides allantoïdiens et/ou amniotiques;

$$
\text { - un conceptus (échogène) }
$$

correspondant à l'embryon ou au fœetus;

- les enveloppes placentaires
(échogènes);

- les cotylédons (échogènes).

Tout comme les autres tests de diagnostics de gestation, les résultats du diagnostic à l'échographie sont évalués selon les critères habituellement utilisés en épidémiologie animale. Il s'agit essentiellement de la précocité, de l'exactitude et de la fiabilité. La précocité facilement défini, vise à classer le test selon le délai auquel on peut y avoir recours à partir du jour d'insémination. L'exactitude a été définie comme l'aptitude à détecter les femelles gestantes ou non gestantes. L'exactitude des diagnostics positifs (en pourcentage) a été calculée comme le rapport du nombre de la situation 'a' sur la somme des nombres de la situation ' $a$ ' et de la situation 'd'. L'exactitude des diagnostics négatifs (en pourcentage) a été calculée comme le rapport du nombre de la situation 'c' sur la somme des nombres de la situation 'c' et de la situation 'b'. L'exactitude globale (en pourcentage) a été calculée comme le rapport de la somme des situations ' $a$ ' et 'c' sur le nombre total des femelles diagnostiquées. La fiabilité a été définie comme la probabilité qu'une femelle déclarée gestante puisse mettre bas ou qu'une femelle déclarée non gestante soit vide. La fiabilité des diagnostics positifs (en pourcentage) a été calculée comme le rapport du nombre de la situation 'a' sur la somme des nombres de la situation 'a' et de la situation 'b'. La fiabilité des diagnostics négatifs (en pourcentage) a été calculée comme le rapport du nombre de la situation 'c' sur la somme des nombres de la situation 'c' et de la situation 'd'.

a : le diagnostic de gestation s'est révélé exact (vrai positif);

b: le diagnostic de gestation s'est révélé inexact (faux positif) ;

c : le diagnostic de non gestation s'est révélé exact (vrai négatif);

$\mathrm{d}$ : le diagnostic de non gestation s'est révélé inexact (faux négatif).

\section{Analyses statistiques}

Les résultats ont été exprimés en moyenne plus ou moins écart-type et les différences considérées comme significatives 
au seuil de probabilité de $5 \%(\mathrm{P}<0,05)$. Les résultats ont été analysés au moyen du logiciel Statistica 6.0.

\section{RÉSULTATS ET DISCUSSION \\ Suivi de la croissance folliculaire par échographie à l'œestrus induit}

Le follicule apparaît sur l'écran de l'échographe comme un disque plus ou moins circulaire anéchogène (noir) limité par une paroie échogène mince (Fgure 1). L'échographie sous évalue de 2 à $3 \mathrm{~mm}$ environ par rapport au diamètre anatomique des follicules car seule la cavité folliculaire est visible à l'écran. Cependant, l'examen échographique prédit bien la taille des follicules car il existe une forte corrélation positive $(R=0,90)$ entre les mesures échographiques et celles en post-morten pour les follicules de taille supérieure à $4 \mathrm{~mm}$ (Driancourt et al., 1988).

La Figure 2 montre une courbe de croissance folliculaire jusqu'à l'ovulation après un traitement de synchronisation et d'ovulation par les progestagènes associés à la Pregnant Mare Serum Gonadotropin (PMSG) chez les vaches Goudali (Pilala et al., 2004). La taille maximale atteinte par les follicules pré-ovulatoires chez les zébus 'Goudali' après un traitement de synchronisation et d'ovulation par les progestagènes associés à la Pregnant Mare Serum Gonadotropin (11,80 \pm $0,96 \mathrm{~mm}$ ) est comparable à la valeur de $10,8 \pm$ $0,7 \mathrm{~mm}$ enregistrée sur les génisses de race zébu Sistani en Iran sans traitement hormonal (Niasari-Naslaji et al., 1999). Cette valeur est également semblable à celles rapportées par Viana et al. (2000) chez les vaches zébus Gir ayant présentées quatre vagues de croissance folliculaire. Ces auteurs ont observé une taille maximale de 10,8 $\pm 2,5 \mathrm{~mm}$ pour le follicule ovulatoire de la première vague ; $9,8 \pm 1,0 \mathrm{~mm}$ pour la deuxième vague ; $9,0 \pm$ $0,8 \mathrm{~mm}$ pour la troisième vague et enfin $13,3 \pm 1,0 \mathrm{~mm}$ pour la quatrième vague. Toutefois, la valeur moyenne estimée reste inférieure à celle des follicules pré-ovulatoires observée chez les bovins Bos taurus européens, laquelle varie entre 15 et $20 \mathrm{~mm}$ (Pierson et Ginther, 1988; Calais et Dreno, 2004). Les différences de taille des follicules pré-ovulatoires observées chez les zébus et les races taurines européennes semblent être liées à différents facteurs tels que l'état nutritionnel (Roche et Boland, 1991) et surtout la taille des ovaires de la femelle zébu, rapportés comme étant plus petits que ceux des taurins européens (Agba, 1975 cité par Pitala, 2005).

L'ovulation est indirectement mise en évidence par la disparition du follicule préovulatoire entre deux examens échographiques successifs, c'est-à-dire entre une image anéchogène d'un follicule préovulatoire et une image échogène correspondant au corps hémorragique (Figure 3). Ceci nous a permis de déterminer par échographie chez les vaches zébus 'Goudali', l'intervalle entre le début des chaleurs et l'ovulation qui se situe entre 22 heures et 26 heures. Ces résultats sont similaires à ceux obtenus par Thiam (1989) chez le zébu Gobra (25,6 $\pm 2,8$ heures après le début des chaleurs) et par Mukasa-Mugerwa et Mattoni (1988) chez le zébu éthiopien $(25,8$ heures après le début des chaleurs). Ces données sont également similaires à celles de Pinheiro et al. (1998) qui ont obtenu une moyenne de 26,6 \pm 0,4 heures chez les femelles zébus Nelore sur œstrus induit ou naturel. Par contre, cette valeur est inférieure à l'intervalle de 28 heures à 32 heures après le début des chaleurs obtenu sur des races taurines européennes selon la littérature.

\section{Diagnostic et suivi de la gestation par échographie}

Le diagnostic de gestation est établi sur la base de la reconnaissance des images correspondant aux liquides contenus dans les vésicules embryonnaires et/ou embryons. L'observation d'une zone anéchogène plus ou moins circulaire dans l'utérus (vésicule embryonnaire) est faite à partir du $28^{\text {ème }}$ jour post-insémination artificielle. Cette période est similaire à celle obtenue par Tainturier et al. (2003). La précocité du diagnostic de gestation dépend des particularités du 
développement du blastocyte, de la parité de la femelle et du pouvoir de résolution de la sonde. Les résultats du diagnostic de gestation chez les femelles zébus Goudali réalisés par Pitala (2005) corroborent les observations rapportées par Chaffaux et al. (1986), lesquels ont détecté la présence des vésicules embryonnaires à partir du $35^{\text {ème }}$ jour de gestation. En utilisant une sonde de $5 \mathrm{MHz}$, Curran et al. (1986) ont pu détecter la présence de la vésicule embryonnaire plus précocement, soit $12,7 \pm 0,4$ jours après insémination, avec des extrêmes allant du $11^{\text {ème }}$ au $18^{\text {ème }}$ jour en faisant des diagnostics quotidiens. En effet, l'accumulation de liquides à l'intérieur de l'utérus peut aussi être observée en absence de gestation, notamment pendant la période de l'œstrus et au début de la phase lutéale ainsi qu'au cours de différents états pathologiques de l'appareil génital telles que les métrites (Chastant-Maillard et al., 2002 ; DesCôteux et al., 2006). Il est utile en cas de doute de vérifier par échographie le caractère fonctionnel du corps jaune sur l'ovaire ipsilatéral ou de rechercher la présence de l'embryon. Etant donnée la précocité du diagnostic de gestation par échographie, il sera préférable, dans le cas d'un diagnostic de gestation précoce, d'échographier à nouveau la vache après 35 jours post-insémination naturelle ou artificielle car à cette date, la vésicule embryonnaire est plus facilement mise en évidence. Mais il est important de pouvoir faire le diagnostic différentiel entre la gestation précoce et une mortalité embryonnaire, un kyste ovarien, un follicule, des vaisseaux utérins, un pyomètre, une métrite et la vessie. Les éléments de ce diagnostic ont été exposés dans une étude faite par Chastant-Millard et al. (2003) (cité par Calais et Dreno, 2004).

L'embryon, tache échogène au sein de la zone anéchogène, a été détecté à partir du $40^{\text {ème }}$ jour post-insémination artificielle (Figure 4). Ce temps est tardif par rapport à une étude réalisée par Lebastard (1997) qui est de 30 jours post-insémination. Curran et al. (1986) ont pu détecter l'embryon dans la corne ipsilatérale au corps jaune dès $21,3 \pm 0,3$ jours post-insémination, avec des extrêmes allant de 20 à 24 jours. Une observation de l'embryon (entre 27 et 30 jours après insémination) a été décrite par Pierson et Ginther (1984) et par Fissore et al. (1988) au moyen d'une sonde de $5 \mathrm{MHz}$.

En fonction de la rotation de la sonde et de la position du foetus, il est possible de visualiser certains organes (cordon ombilical) et structures osseuses fœtales (crâne), à partir $\mathrm{du} 45^{\text {ème }}$ jour de gestation (Figure 5).

L'interprétation des critères de qualité de diagnostic de gestation par échographie dépend du stade de gestation auquel le diagnostic a été posé, des critères de diagnostic de gestation retenus, de la fréquence d'émission de la sonde échographique, de la fréquence de la mortalité embryonnaire précoce ou tardive, ainsi que de l'âge de l'animal. Ainsi, l'exactitude globale de l'échographie transrectale utilisée au cours du présent travail est passée de $92,2 \%$ à $98,4 \%$ entre le $28^{\text {ème }}$ et le $40^{\text {ème }}$ jour postinsémination. Ceci confirme le fait que plus on avance dans la gestation, plus la vésicule amniotique et/ou l'embryon sont faciles à détecter. Ces résultats sont conformes aux observations rapportées par différents auteurs (Filteau et DesCôteaux, 1998 ; DeCôteaux et al., 2006), lesquels ont décrit une exactitude de 92 à $98 \%$ au-delà de 30 jours de gestation. Par contre, nos résultats sont légèrement inférieurs à l'exactitude globale de 100\% atteinte à partir du $35^{\text {ème }}$ jour de gestation chez des vaches d'origine européenne âgées de moins de 7 ans (Hugues et Davies, 1989; Hanzen et Laurent, 1991) et ayant eu moins de 7 vêlages (Hugues et Davies, 1989).

Dans une étude réalisée en 1995, Szenci et collaborateurs ont montré qu'après le $24^{\text {ème }}$ jour de gestation, plus l'utérus est en position crâniale par rapport à l'os pelvien, plus on risque de ne pas détecter l'embryon (faux négatif). Les cas douteux observés pendant le premier mois de gestation pourraient aussi être attribués au fait que pendant cette période l'utérus a une forme plus tortueuse, ce qui le rend plus difficile à 
être balayé systématiquement par la sonde transrectale de l'échographe (Szenci et al., 1995). En outre, Pieterse (1998), a démontré que c'est seulement à partir du $25^{\text {ème }}$ jour, que les vésicules contenant des liquides amniotiques deviennent plus faciles à distinguer à l'intérieur de l'utérus bovin. Il faut également noter que l'un des facteurs pouvant affecter la précision du diagnostic de gestation est sans doute le moment de la gestation où l'examen reproducteur est effectué. La fiabilité du diagnostic de gestation négatif obtenu au cours de cette étude expérimentale a augmenté de $94,4 \%$ au $28^{\text {ème }}$ jour à $100 \%$ au $40^{\text {ème }}$ jour, ce qui indique que toutes les vaches zébus diagnostiquées vides au $40^{\text {ème }}$ jour postinsémination ont été réellement non gravides. Ces résultats sont légèrement supérieurs à ceux décrits par Szenci et al. (1998), lesquels ont obtenu une fiabilité de $95,3 \%$ et de $98,3 \%$, respectivement pour le $39^{\text {ème }}-42^{\text {ème }}$ et pour les 44 ème-45 ème jours de gestation. En effet, dans leurs études, Hanzen et al. (1993) ont comparé les résultats de différentes études publiées sur la fiabilité des diagnostics de gestation par échographie. Globalement, cette
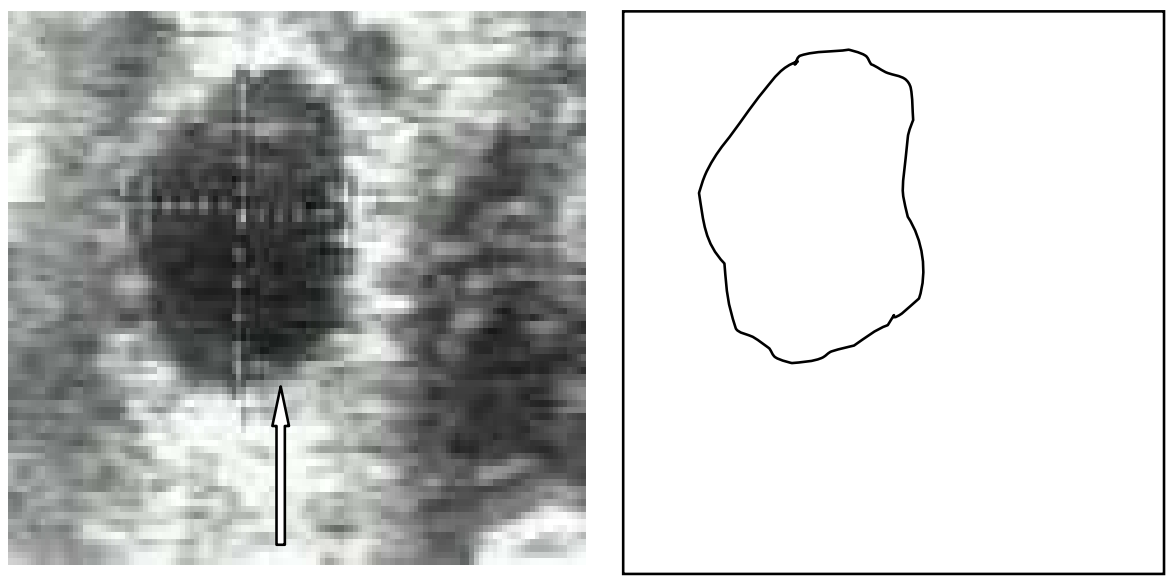

Follicule pré-ovulatoire étude montre que l'échographie est plus apte à détecter les animaux gestants (sensibilité : 91\% (51-99)) que non-gestants (spécificité : $74 \%$ (74-95)). Le degré d'exactitude des diagnostics de gestation (91\% (70-99)) est supérieur à celui des diagnostics de nongestation $(80 \%$ (57-97)). Le degré d'exactitude totale est de $87 \%$ (63-98).

Plusieurs études ont été consacrées à la croissance et à la détermination de l'âge du fœtus bovin par la mesure de ses structures anatomiques (Kähn, 1989; Laigre et al., 2004 ; Chavatte-Palmer et al., 2006; KohanGhardr et al., 2008). A partir du $120^{\text {ème }}$ jour de gestation, la taille et la position de l'utérus ne permettent pas le balayage systématique en entier du fotus par la sonde, ce qui empêche la mensuration des différentes parties des fœtus. Durant cette période, le fœtus est très éloigné de la sonde, on ne peut alors observer que de volumineux cotylédons et un utérus contenant une grande quantité de liquide (Figure 6). Mais Le Cleac'h (2011) a pu étudier la croissance chez les fotus clonés au delà de 150 jours de gestation par échographie transabdominale.

Figure 1: Image échographique d'un ovaire d'une vache zébu 'Goudali' présentant un follicule préovulatoire (dominant) de forme plus ou moins sphérique 44 heures après la fin du traitement de synchronisation et d'ovulation associant les progestagènes et la PMSG (Pitala et al., 2004). 


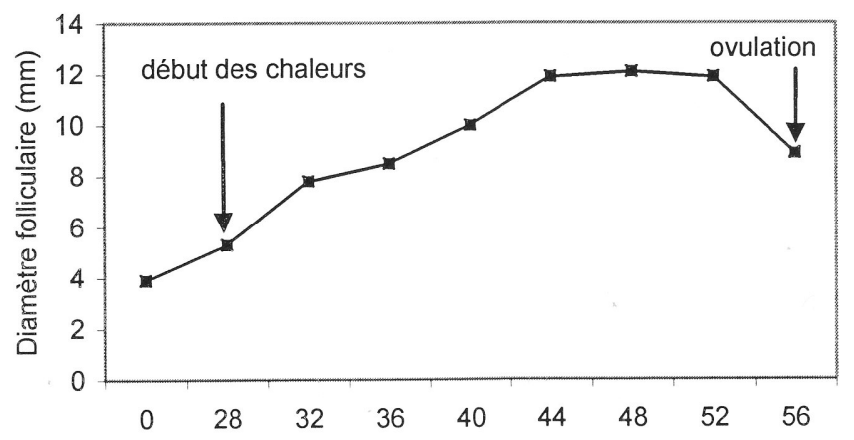

Figure 2: Dynamique de la de croissance de follicule dominant chez les vaches zébus 'Goudali' entre la fin du traitement et l'ovulation constatée (Pitala et al., 2004). Temps après le retrait des implants (heures).

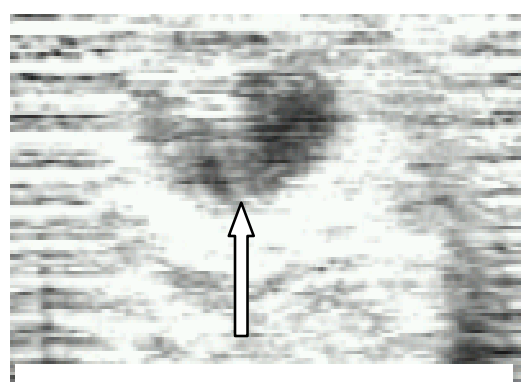

Corps jaune en formation

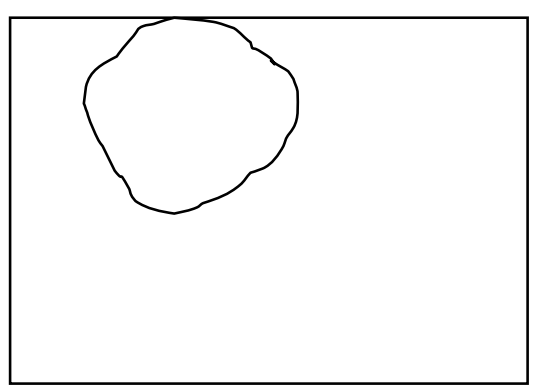

Figure 3: Image échographique d'un ovaire d'une vache zébu 'Goudali' le jour de l'ovulation montrant un corps hémorragique (Pitala et al., 2004).

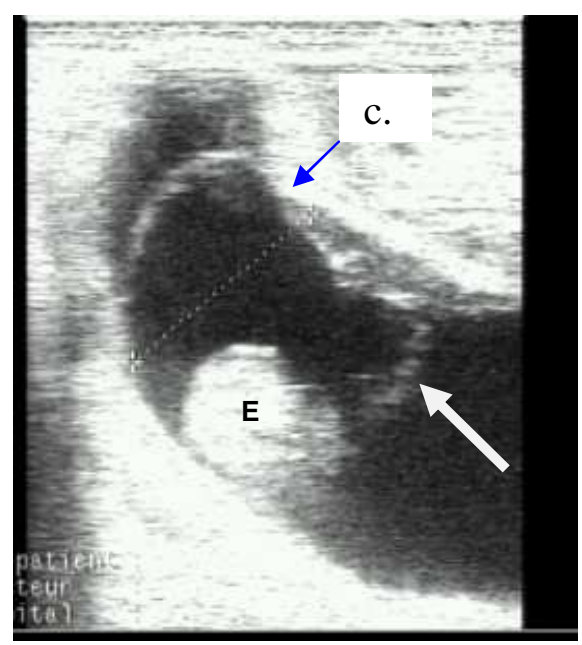

Figure 4: Image échographique chez une vache Goudali gestante de 40 jours post-insémination. E : indique l'embryon très échogène. c.u : corne utérine contenant le liquide amniotique très anéchogène; flèche blanche : membrane amniotique (Pitala et al., 2003). 

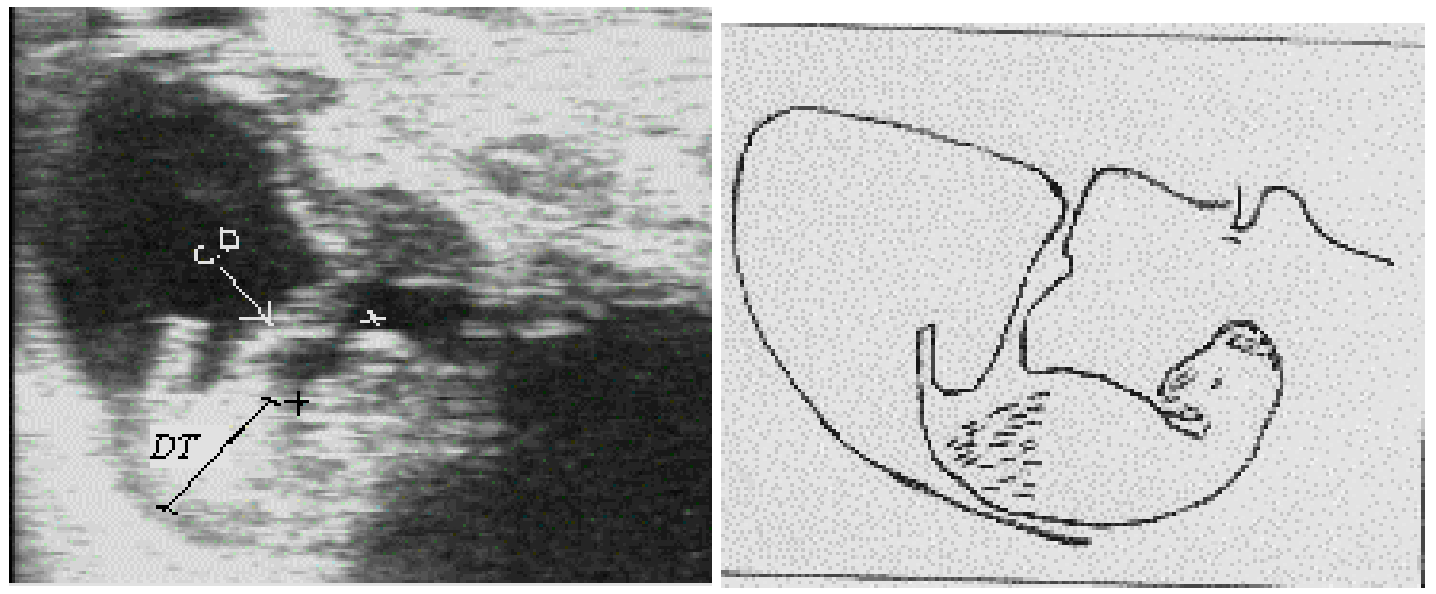

Figure 5: Image échographique d'un fœus 45 jours post-insémination d'une vache Goudali. c.o. indique le cordon ombilical ; + indique la délimitation du crâne (Pitala et al., 2003).

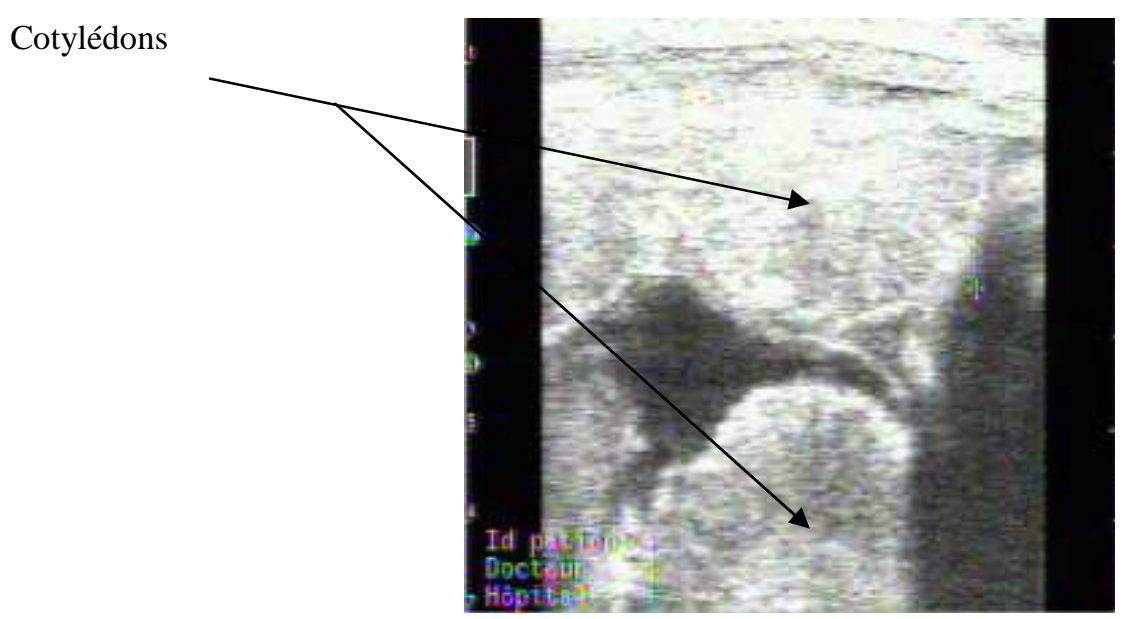

Figure 6: Image échographique d'une gestation de 120 jours montrant les cotylédons anéchogènes (Pitala, 2005).

\section{Conclusion}

Comme un outil de recherche appliquée, l'échographie transrectale a apporté des innovations importantes dans le domaine de la reproduction des bovins en particulier. La dynamique de la croissance folliculaire du zébu Goudali a pu être précisée grâce à l'échographie transrectale munie d'une sonde linéaire de $6 \mathrm{MHz}$. Elle a permis de mieux prévoir le moment optimum d'ovulations (52 à 56 heures après la fin du traitement) et la taille des follicules dominants $(11,80 \pm 0,96 \mathrm{~mm})$. La dynamique de la gestation a fait également l'objet de notre étude. L'échographie transrectale avec une sonde de $6 \mathrm{MHz}$ est à la fois précoce ( $28^{\text {ème }}$ jour post insémination) et fiable car elle permet de diagnostiquer en moyenne $89,3 \%$ et de $94,4 \%$ respectivement 
pour les animaux gestants et non-gestants avec une exactitude globale de $92,2 \%$ au $28^{\text {ème }}$ jour. Elle a aussi permis l'étude du développement morphologique de la vésicule embryonnaire, de l'embryon, du fœtus et le diagnostic des malformations fœtales. De nos jours il existe plusieurs méthodes d'étude de la physiologie ovarienne et de diagnostic de gestation chez la vache: les méthodes dites de laboratoire reposant sur la mesure des concentrations des hormones et les méthodes dites cliniques comprenant la palpation transrectale et l'échographie. Comparativement aux méthodes de laboratoire, l'échographie donne des résultats immédiats. Le dosage de la progestérone, le dosage des protéines placentaires et l'échotomographie sont toutes des techniques précoces de gestation, mais l'échotomographie est la seule méthode qui donne les résultats sans délai.

Cette étude a permis de souligner des inconvénients et des limites de l'échographie. En effet, l'échographie ne donne aucun renseignement sur la qualité de l'ovocyte qui est l'une des conditions indispensables de la fécondation.

\section{REFERENCES}

Boly H, Coulibaly I, Pitala W, Ba Y, Sawadogo L, 2003. Comportement sexuel et maturation folliculaire chez la femelle zébu Goudali au Burkina Faso. Revue Africaine de Santé et des Productions Animales, 1: 102-107.

Calais EIM, Dreno CM. 2004. Echographie en gynécologie bovine, ovine et caprine : réalisation de CD-ROM didactique. Thèse de Doctorat Vétérinaire, Ecole Nationale Vétérinaire d'Alfort, 215p.

Chaffaux S, Bianci M, Hedge GV, Reddy GNJ, Thibier M. 1986. L'échographie en temps réel par voie transrectale : intérêt pour le diagnostic de gestation chez la vache. Rec. Méd. Vét., 164: 101-108.

Chastant-Maillard S, Boin E, Constant F, Mialot JP, Grimard B, Druart X. 2002. Images échographiques de la gestation chez la vache. Gestation. In Journées
Européennes de la Société Française de Buiatrie, Paris, France, 29-31 octobre 2002, p. 186-195.

Chavatte-Palmer P, De Sousa N, Laigre P, Camous S, Ponter AA et Beckers JF. 2006. Ultrasound fetal measurements and pregnancy associated glycoprotein secretion in early pregnancy in cattle recipients carrying somatic clones. Therigenelogy, 66: 829-840.

Cros N, 2005. Le sexage des fœtus par échographie chez la vache: étude de l'utilisation pratique sur le terrain. Thèse de Doctorat vétérinaire de l'école vétérinaire de Lyon, 159p.

Curran S, Pierson RA, Ginther OJ. 1986. Ultrasonographic appearance of the bovine conceptus from days 10 through 20. J. Am. Vet. Med. Assoc., 189: 12891294.

DesCôteaux L, Carrière PD, Drocher J. 2006. Ultrasonography of the reproductive system of the cow: basic principles, pratical sues and economic aspects of this diagnostic tool in dairy production. $\mathrm{XXIV}^{\text {th }}$ world buiatric congress 2006, Nice-France consulté sur le site www:ivis.org le 27 septembre 2012.

Driancourt MA, Andrieu D, Thatcher WW. 1988. Suivi de la croissance folliculaire par échographie au cours des cycles naturels et stimulés chez la vache. Recueil de Médécine Vétérinaire, 164: 109-114.

Filteau V, DesCôteaux L. 1998. Valeurs prédictives de l'utilisation de l'appareil échographique pour le diagnostic précoce de gestation chez la vache laitière. Le Médecin Vétérinaire du Québec, 28: 8185.

Fissore RA, Edmondson AJ, Pashen RL, Bondurant RH. 1988. The use of ultrasonography for the study of the bovine reproductive tract. I Normal and pathology ovarian structures. Anim. Reprod. Sci., 12: 167-177.

Francony SM. 2011. Comparaison de l'intérêt diagnostique des différentes techniques d'examen de l'ovaire de la vache. Thèse de doctorat vétérinaire, Ecole Nationale 
Vétérinaire d'Alfort, 135p.

Hanzen C, Laurent Y. 1991. Application de l'échographie bidimensionnelle au diagnostic de gestation et à l'évaluation de l'incidence de la mortalité embryonnaire dans l'espèce bovine. Annales de Médecine Vétérinaire, 135: 481-487.

Hanzen C, Laurent Y, Jakovljvics S, 1993. Applications de l'échographie en reproduction bovine. 2. L'utérus gestant et non-gestant. Ann. Méd. Vét., 137: 93101.

Hugues EA, Davies DAR. 1989. Practical uses of ultrasound in early pregnancy in cattle. Vet. Rec., 124: 456-458.

Kähn W. 1989. Sonographic fetometry in bovine. Theriogenology, 31: 1105-1121.

Kohan-Ghardr HR, Lefebvre RC, Fecteau G. 2008. Ultrasonography and histological characterizationof the placenta of somatic nuclear transfer-derived pregnancy in dairy cattle. Theriogenology, 69: 218230.

Laigre P, Chavatte-Palmer P, Vignon X, et Heyman Y, 2004. Suivi échographique de gestations bovines après transfert d'embryons clonés. Renc. Rech. Rum., 11: 393.

Lebastard D. 1997. Echographie en gynécologie bovine : utilisations possibles dans le cadre d'un exercice en clientèle rurale. Point Vét., 28: 10891096.

Le Cleac'h NLP. 2011. Evaluation du développement placentaire et fœtal de gestations des clones bovins par échographie transabdominale à partir de 150 jours de gestation. Thèse de médecine vétérinaire de Nantes, 116p.

Mialot JP, Chastant-Maillard S, Boin E, Constant F, Grimard B. 2002. Imagerie échographique de l'utérus et des ovaires en gynécologie bovine. In Journées Nationales des GTV, Tours, pp 679-684.

Niasari-Naslaji A, Sarhaddi F, Naji A, Angurani A, Damvandi Y. 1999. Ovarian follicular dynamics in Bos taurus and Bos indicus. Theriogenology, 51: 307.
Mukasa-Mugerwa E, Mattoni M. 1988. Estrous behavior in zebu cattle. IFS/SIPAR Joint Seminar on Animal Reproduction for African Countries Addis Ababa, 56-64.

Pierson RA, Ginther OJ. 1984. Ultrasonography for detection of pregnancy and study of embryonic development in heifers. Theriogenology, 22: 225-233.

Pierson RA, Ginther OJ. 1988. Follicular populations during the estrous cycle in heifers III: Time of selection of the ovulatory follicle. Anim. Reprod. Sci., 16: 81-96.

Pieterse C M, 1998. Echographie en reproduction bovine (utérus et ovaires: diagnostic et traitement). Le nouveau peripartum. Congrès de la Société Française de Buiatrie, Paris, France, 2526 novembre 1998, 221-228.

Pinheiro OL, Barros CM, Figueiredo RA, Valle ER, Encarnça RO, Padovani CR. 1998. Estrous behavior and the estrus-toovulation interval in Nelore cattle (Bos indicus) with natural estrus or estrus induced with prostaglandin $\mathrm{F} 2 \alpha$ or norgestomet and estradiol valerate, Theriogenology, 49: 667-681.

Pitala W, 2005. Dynamique folliculaire et suivi de la gestation par échographie chez le zébu Goudali au Burkina Faso. Thèse de doctorat d'université, Université de Ouagadougou, 109p.

Pitala W, Boly H, Zongo M, Ba Y, Sousa N M, Sawadogo L, Leroy P, Beckers JF, 2003. Diagnostic et suivi de la gravidité chez le zébu par échographie. Revue Elev. Méd. Vét. Pays Tropicaux, 56: 171-176.

Pitala W, Boly H, Zongo M, Coulibaly I, Sousa NM , Sawadogo L, Leroy P, Beckers JF. 2004. Application de l'échographie à l'étude de la dynamique folliculaire à l'œstrus induit chez les femelles zébu 'Goudali'. Tropicultura, 22: 110-115.

Quirk SM, Hickey GJ, Fortune JE. 1986. Growth and regression of ovarian follicles during the follicular phase of the 
estrus cycle in heifers undergoing spontaneous and PGF2 $\alpha$-induced luteosis. J. Reprod. Fert., 77: 211-219.

Roche JF, Boland MP. 1991. Tumover of dominat follicles in cattle different reproductive. Theriogenology, 35: 81-90.

Szenci O, Beckers JF, Humblot P, Sulon J, Sasser G, Taverne MAM, Varga J, Baltusen R, Schekk GY. 1998. Comparison of ultrasonography, bovine pregnancy-specific protein $\mathrm{B}$, and bovine pregnancy-associated glycoprotein 1 tests for pregnancy detection in dairy cows. Theriogenology, 50 : 77-88.

Szenci O, Gyalai GY, Nagy P, Lovacs L, Varga J, Taverne MAM, 1995. Effect of uterus position to de pelvic inlet on the accuracy of early bovine pregnancy diagnosis by means of ultrasonography. Vet. Quarterly, 17: 37-39.

Thiam MM. 1989. Actualité sue la maîtrise du cycle sexuel chez la femelle zébu (Bos indicus) en Afrique. Th. Méd. Vét. Dakar, p. 14.

Taiturier B, Taiturier D, Bencharif D, 2003. Sexage précoce du fœtus par échographie chez la vache. Le Point Vétérinaire, 34: 106-110.

Viana JAM, Ferreira ADM, Sa WF, Camargo LSA. 2000. Follicular dynamics in zebu cattle. Pesq. Agropec. Bras., 35: 25012509. 